\title{
A STRUCTURED APPROACH TO REDUCE DESIGN ITERATIONS IN ADDITIVE MANUFACTURING
}

\author{
Wirths, Laura (1); \\ Bleckmann, Matthias (2); \\ Paetzold, Kristin (1) \\ 1: Bundeswehr University Munich; \\ 2: Bundeswehr Research Institute for Materials, Fuels and Lubricants (WIWeB)
}

\begin{abstract}
Additive Manufacturing technologies are based on a layer-by-layer build-up. This offers the possibility to design complex geometries or to integrate functionalities in the part. Nevertheless, limitations given by the manufacturing process apply to the geometric design freedom. These limitations are often unknown due to a lack of knowledge of the cause-effect relationships of the process. Currently, this leads to many iterations until the final part fulfils its functionality. Particularly for small batch sizes, producing the part at the first attempt is very important. In this study, a structured approach to reduce the design iterations is presented. Therefore, the cause-effect relationships are systematically established and analysed in detail. Based on this knowledge, design guidelines can be derived. These guidelines consider process limitations and help to reduce the iterations for the final part production. In order to illustrate the approach, the spare parts production via laser powder bed fusion is used as an example.
\end{abstract}

Keywords: 3D printing, Additive Manufacturing, Design for Additive Manufacturing (DfAM), Process modelling, Design engineering

\author{
Contact: \\ Wirths, Laura \\ Bundeswehr University Munich \\ Department of Aerospace Engineering \\ Germany \\ laura.wirths@unibw.de
}

Cite this article: Wirths, L., Bleckmann, M., Paetzold, K. (2021) 'A Structured Approach to Reduce Design Iterations in Additive Manufacturing', in Proceedings of the International Conference on Engineering Design (ICED21), Gothenburg, Sweden, 16-20 August 2021. DOI:10.1017/pds.2021.24 


\section{INTRODUCTION}

Additive manufacturing (AM) technologies provide the opportunity to realise parts with designs and features that were not possible with traditional processes. The part design is an essential process step to implement such designs and features. To realise these new opportunities, conventional design guidelines cannot be applied to the new process and have to be adapted for the additive manufacturing processes. Only in this way, the potential of the manufacturing process can be fully exploited.

Additive manufacturing technologies, such as the Laser Powder Bed Fusion (L-PBF) process for metals, are based on a layer-by-layer build-up. In this process, a uniform layer of powder is applied on a build plate and subsequently molten by a laser to form a solid compound. The laser paths are based on a CAD model, which is previously divided into defined layers (slicing). After one layer of the component has been completely scanned by the laser, the build plate is lowered by the layer height. Then the next powder layer is applied. In this way, the complete part is produced layer-by-layer.

Based on this manufacturing approach, the L-PBF process is promised the greatest possible geometric design freedom. Several examples, demonstrating the possible complexity of geometries are given in literature. Some examples are lightweight structures for the aerospace industry (Kranz et al., 2015), internal structures such as cooling channels (Shinde and Ashtankar, 2017) or individualized product design for prostheses, body protectives or sport equipment (Beiderbeck et al., 2020). The new design opportunities enable the combination of several individual components into a single one by means of integral construction. An example taken from the automotive industry is a wheel carrier with an integrated brake calliper (Fraunhofer IAPT, 2020).

Although the layer-wise manner of the production offers many opportunities for the part design, yet there are still limitations given by the process. Such limitations are often neglected by unexperienced personnel, which in turn leads to non-fulfilment of the desired part functionality. Only with increasing experience, these limitations are taken into account and the potential of the manufacturing technology can be exploited.

One obvious example for a limitation that cannot be realised by L-PBF is entirely enclosed hollow volumes. In this case, holes have to be considered in the part design, so that the internal unmelted powder can be removed. A further example that is already well discussed and is showing the evaluation of design rules is overhanging surfaces. As a rule of thumb, overhang angles greater than 45 degrees in respect to the vertical have to be avoided or supported by support structures (Diegel et al., 2019; Thomas, 2009). As manufacturing technologies improve, such rules for the part design are continuously in motion and have to be adjusted. For example, some approaches already show that unsupported overhangs are even possible down to 30 degrees (Di Wang et al., 2013; Mertens et al., 2014).

However, to tap the full potential of such manufacturing processes, it is necessary to identify the limitations. Especially for the spare part production as well as single part production, it is important to successfully produce components on the first attempt. However, this goal has not yet been achieved. Especially with more complex components, the first attempt often does not reach the requirements. The cause is that numerous factors have an impact on the success of the print and the fulfilment of the requirements. These factors can even lead to an abrupt termination of the manufacturing process. This finally leads to several necessary iterations. In each iteration loop, small changes are made, which in most cases are based on experience or are carried out according to the trial-and-error approach. This approach is used in new manufacturing processes mainly because there is a lack of knowledge about the cause-effect relationships. To reduce the number of iterations right from the start, it is necessary to study intensively the factors, which can occur during the manufacturing process. From these findings, limitations can be derived, which are given by the manufacturing technology. Such limitations have to be addressed by new or adapted design guidelines. In this way, the possibilities and limitations can be taken into account for manufacturing processes.

Especially in the spare part production, where additive manufacturing is used to replace conventional manufacturing methods, it is of great interest that the number of required iterations is kept to a minimum. This is because with each failed attempt, the profitability of the new manufacturing process with respect to the conventional process decreases. In this study the procedure for determining limitations of a process and addressing them by design guidelines is described in detail using the L-PBF spare part production as an example. Therefore, the following research questions were derived: 
$R Q 1:$ How can cause-effect relationships be systematically identified in a complex system?

$R Q 2$ : How can this knowledge from the cause-effect relationships be utilised to reduce iterations in spare part production via L-PBF?

\section{PROCESS FOR SPARE PART PRODUCTION VIA ADDITIVE MANUFACTURING}

The prime aim in the production of spare parts is to ensure that the functionality of the original part is fulfilled by the spare part. To achieve this goal, the functionality of the original part needs to be specified by requirements. These requirements generally consist of mechanical properties, geometrical properties and additional requirements. The mechanical properties are for example tensile strength, elasticity or hardness, whereas geometric properties include dimensions and surface finish. Additional requirements are aesthetic features or conditions by special application sectors. In the medical sector for example, a material that is used for an implant must have biological properties like biocompatibility, or materials that are used for marine spare parts must have a good corrosion resistance. The determination and establishment of such requirements for the spare part production is described for example by Montero et al. (2020).

After listing the required properties based on the original part, the process defined in Figure 1 is followed. The process consists of five steps: Selection of the manufacturing technology and material, part design, manufacturing process, additional processing, testing the properties.

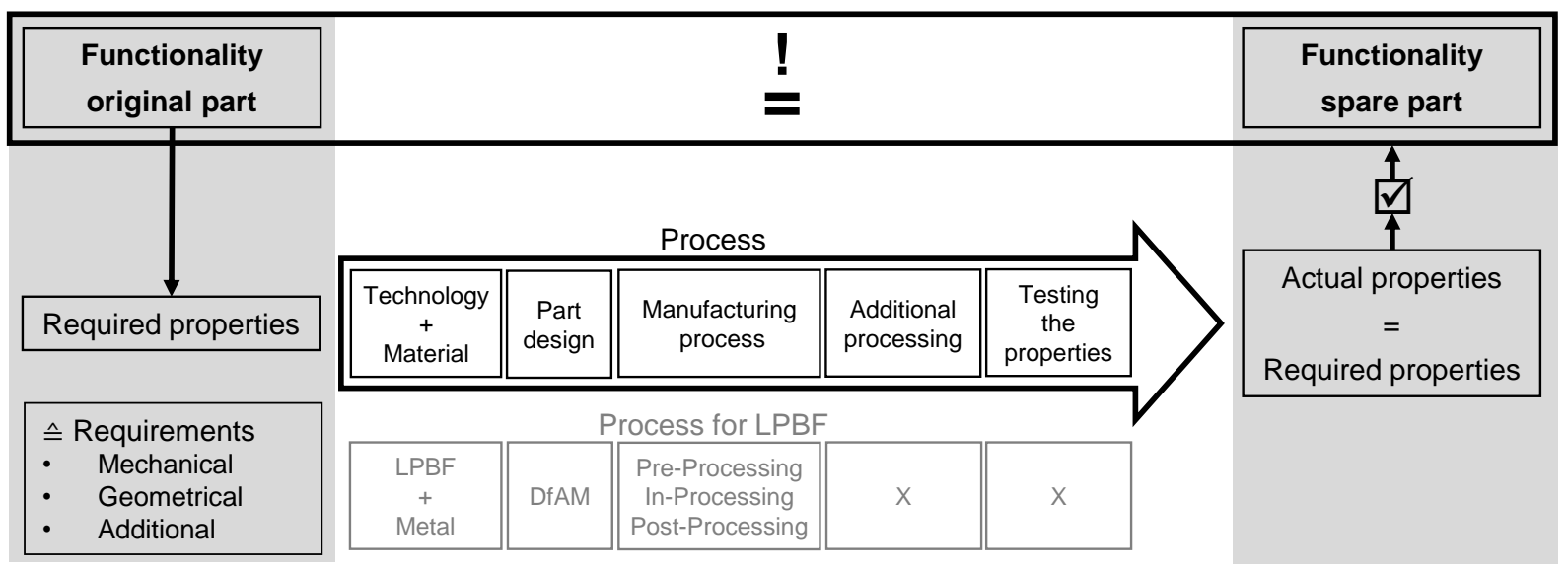

Figure 1. Process for spare part production via additive manufacturing

- Step 1: The first step is the selection of the manufacturing technology and the material. Especially for the production of spare parts with additive manufacturing, there are numerous approaches to determine whether this process is more profitable than the conventional process (Frandsen et al., 2020). In the following, Laser Powder Bed Fusion (L-PBF) is selected as technology and metal as material class.

- Step 2: The second step is the part design. This is influenced by the previously selected manufacturing technology. For the L-PBF technology selected in this case, Design for Additive Manufacturing (DfAM) guidelines for metal should be considered.

- Step 3: In the third step, the spare part is manufactured using the selected technology and material. Depending on the technology, the manufacturing process can be divided into further sub-steps. For L-PBF, this division consists according to DIN SPEC 17071 of pre-processing, in-processing and post-processing. Pre-processing describes all preparations, which are made before the print job starts. This includes for example converting the CAD file into a STL file, placing the part on the software's virtual build plate, orientating the part, slicing, setting of parameters as well as filling the machine with metal powder. In-processing describes the manufacturing process, which is mainly controlled by the process parameters. Post-processing includes additional processing steps after the manufacturing step. For L-PBF, these are the removal of the part from the build plate, cleaning the part from powder residues, removal of support structures and heat treatment. 
- Step 4: In the fourth step, additional processing is carried out, which is necessary for the fulfilment of the required properties and thus for the part's functionality. Processing steps like subtractive machining or surface finishing do not depend explicitly on the manufacturing technology. Which procedures are necessary, depends on the requirements.

- Step 5: In the final step, the required properties, derived from the original part, are checked. Suitable test procedures have to be selected for this. As they depend on the requirements, the test procedures are not further defined here.

In an optimal scenario, the last process step leads to the result that the actual properties of the spare part match the required properties of the original part. In this case, the prime aim of the spare part production is fulfilled and the functionality of the original part is given by the spare part.

\section{Challenges}

The optimum scenario described before, is in most cases only feasible for simple parts with low functional criteria. These parts only have to meet a few requirements. For complex parts, the high requirements often cannot be achieved on the first try. The challenges associated with them are illustrated in Figure 2.

For a complex part, numerous factors affect the process described in section 2. Examples of these factors are unsuitable designs for L-PBF, wrong process parameters or an inadequate part orientation. Such factors can lead to an abrupt termination of the manufacturing process or cause defects in the parts. The consequence of these defects is that the actual properties of the spare part do not match the required properties. To achieve the fully functional part, changes are made in the individual process steps. In most cases, such changes are made based on experience or according to the trial-and-error approach. This leads to a large number of iterations. In contrast to long and extensively used processes such as casting, there is a lack of knowledge of the cause-effect relationships of new processes. Therefore, when a defect occurs, it is difficult to identify the right cause, intervene in the right process step and find the right action to avoid or correct the defect.

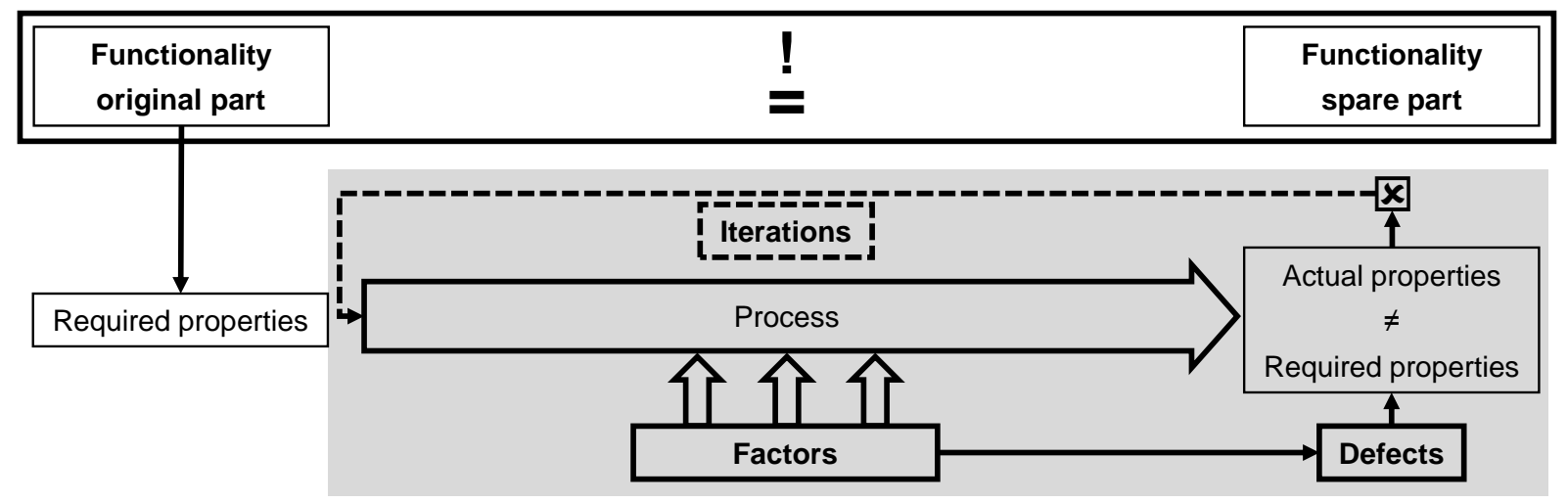

Figure 2. Process with iterations. Influencing factors on the process cause defects. These defects lead to necessary iterations to meet the required properties.

It matters to understand that the individual process steps are related to each other. For example, the choice of process parameters for the manufacturing process depends on the chosen material. Moreover, at the same time, the correlations between the single manufacturing steps have to be considered. Thus, the orientation of the part in the pre-processing influences the removal of support structures in the post-processing. Nevertheless, the part orientation, as a process parameter, can be considered in the part design, so that less removal of support structures will be necessary. Therefore, the part design is as important for AM parts as the process parameters.

It is a challenge to identify cause-effect relationships and to find out the complex correlations within the manufacturing process. For this reason, a structured approach is necessary, in which the entire process is considered. Additionally separate sub-steps are examined in detail in order to subsequently generate solutions. This approach should also help to summarise the large number of results. 


\section{A STRUCTURED APPROACH FOR ITERATION REDUCTION IN SPARE PART PRODUCTION}

The main idea is based on finding solutions with the help of cause-effect relationships, which can be implemented in the design process. This prevents the occurrence of defects in an early stage.

Figure 3 shows the approach and the implemented methods and tools. The first step is the identification of defects, which might occur in the manufacturing process. The impact of the defects on the mechanical and geometrical properties are then determined in the second step. In the third step, the cause-effect relationships are created. Defects are defined as effects and the causes are related to the occurring factors in the process. The next step is to analyse the sub-causes in detail. The results of the analyses are then used to determine solutions that can be implemented in the design process.

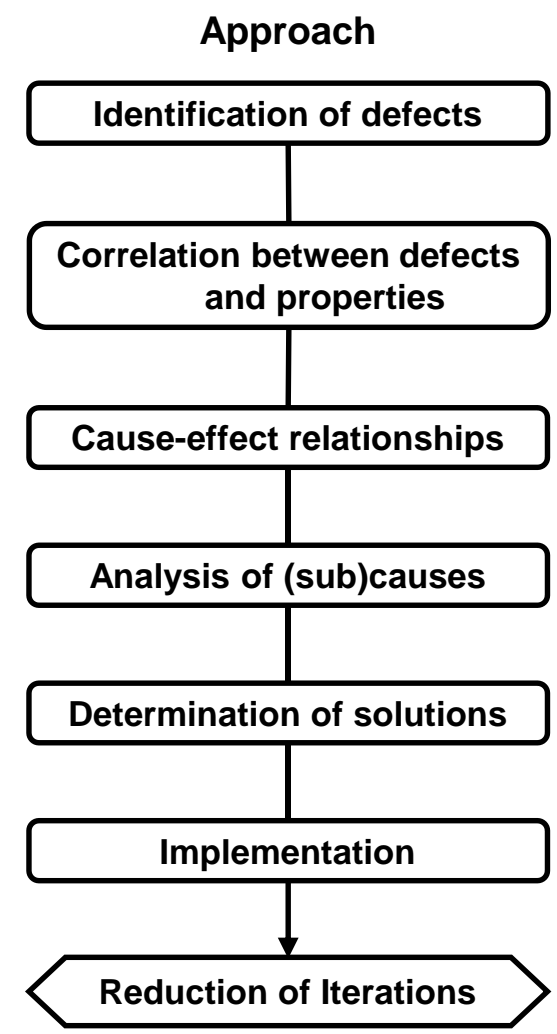

\section{Implemented methods and tools}
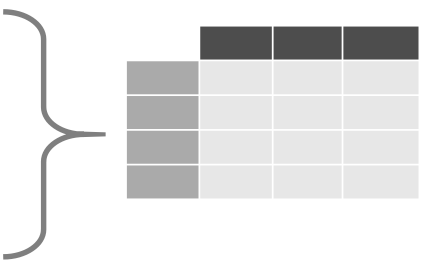

L-shaped

Matrix Diagram

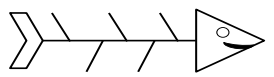

Ishikawa-Diagram

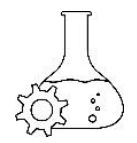

Experiments

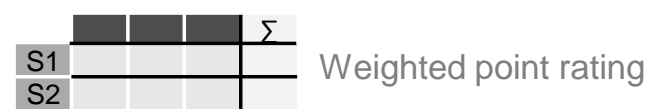

Figure 3. Approach for design iteration reduction in additive manufacturing

In the following sub-sections, the individual steps of the approach are applied to spare part production via L-PBF. Different methods and tools were used to follow the steps or to document the results.

\subsection{Identification of defects and effect on properties}

In the first step, defects were identified which might led to the consequence that the required properties are not fulfilled. Subsequently, the relationships between the type of defect and the properties were elaborated. Based on a literature research, the most frequently occurring defects can be summarised as: (Unintended) porosity, inadequate surface roughness, residual stresses, geometrical deviations, cracks, lack of fusion, anisotropy, bonding errors and delamination.

It should be considered that these defects can also be interdependent. For example, residual stresses can lead to geometrical deviations (Zaeh and Branner, 2010) or surface pores can have an effect on surface roughness (Maamoun et al., 2018). For a well-structured overview of the defects and their influence on the properties, the visualisation in an L-shaped matrix diagram (Jakoby, 2019) is suitable. The first dimension is represented by the defect types and the second dimension by the properties. For example, a reduced cross-section due to porosity has an influence on ultimate tensile strength (Weber et al., 2020b), elongation, Young's modulus and fatigue performance (Stef et al., 2018). Another example of the influence on mechanical properties is surface roughness. Particularly with as-built surfaces, deep valleys in the surface profile lead to stress concentration that promote crack initiation and thus reduce fatigue life (Kasperovich and Hausmann, 2015). In figure 4, exemplarily some 
correlations between defects and properties are summarised in an L-shaped matrix diagram using Excel as a tool.

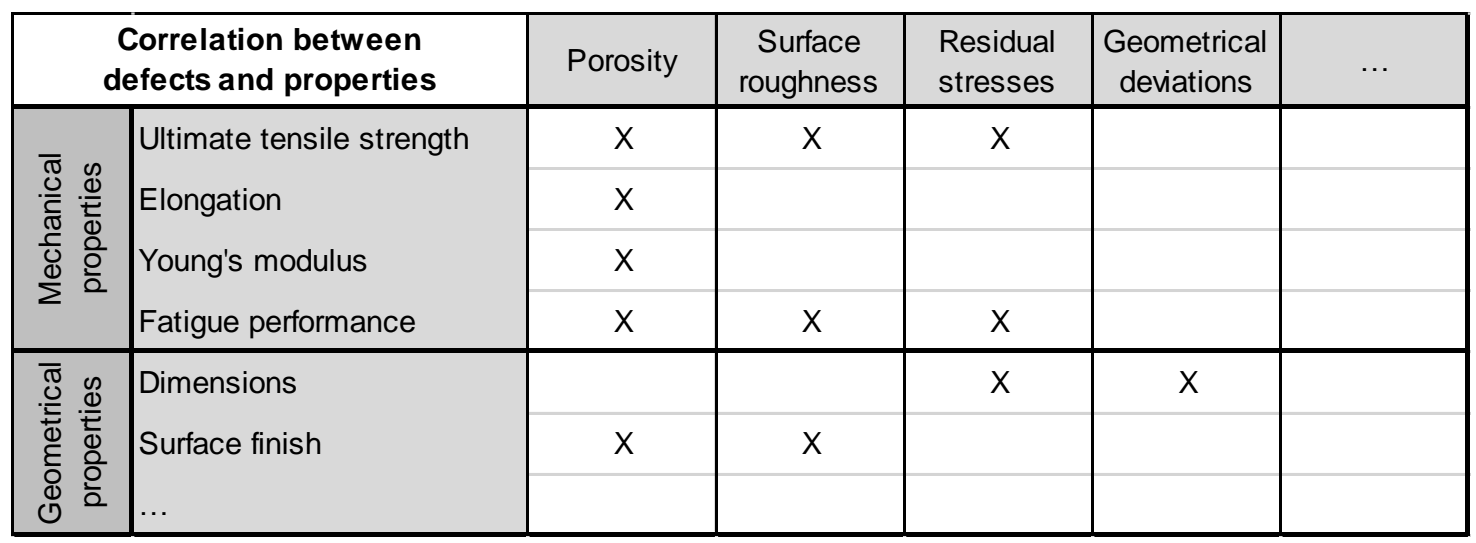

Figure 4. L-shaped matrix diagram for correlations between defects and properties

\subsection{Determination of cause-effect relationships}

After the defects (= effects) have been determined, the causes of each defect must be investigated in detail. For the investigation of such cause-effect relationships, numerous suitable methods already exist which can be applied. Some examples are the Cause and Effect Matrix, the Fault Tree Analysis or the Ishikawa-Diagram, which is also known as fishbone diagram (Jakoby, 2019).

In this study the cause-effect relationships of surface roughness are analysed by the use of the Ishikawa-Diagram. The head of the diagram in figure 5a shows the surface roughness as an effect and the single process steps of the process (for spare part production, as described in section 2) are defined as the main causes. Based on experience and research, further sub-causes are created for the respective main causes. In figure $5 \mathrm{~b}$ for simplicity reasons, only a part of the manufacturing process is shown in detail. Due to the high complexity, the individual main causes should be examined one after another. Nevertheless, all other causes must always be considered in total. XMind was used as a tool for creating the Ishikawa-Diagram. The figure below was drawn manually for readability.

a

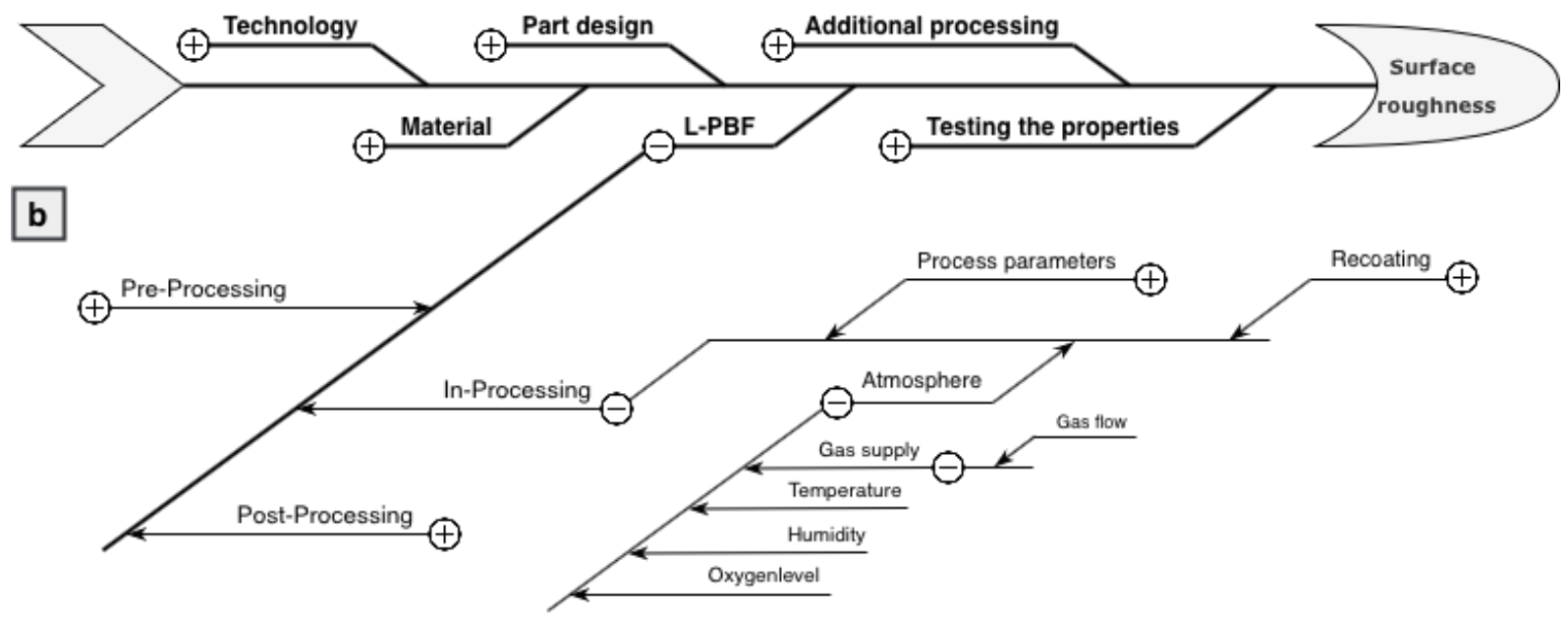

Figure 5. Factors affecting the surface roughness of a spare part manufactured by L-PBF using the Ishikawa-Diagram

\subsection{Analysis of sub-causes}

The Ishikawa-Diagram helps to structure the complex relationships. In most cases however, it is not sufficient to know the qualitative relationship. Therefore, it is necessary to analyse each single subcause in a quantitative way. As an example, a detailed analysis was carried out for the sub-cause gas flow using experiments (Figure 6). 
In the L-PBF process, a protective gas flows through the build chamber to prevent oxidation of the metal. It was assumed that powder particles or spatters are entrained by the gas flow. As a result, a different surface roughness is expected on the gas-facing surfaces than on the gas-averted surfaces. To investigate this in more detail, nine test specimens were printed. The surface roughness was then measured on the gas-facing surface and on the gas-averted surface and compared to each other. It was found that the surface roughness on the gas-averted surfaces $(\mathrm{Sa}=17.1 \pm 1.7 \mu \mathrm{m})$ is higher than on the gas-facing surfaces $(\mathrm{Sa}=14.8 \pm 1.8 \mu \mathrm{m})$. This result agrees with findings from similar investigations

(Li et al., 2018).
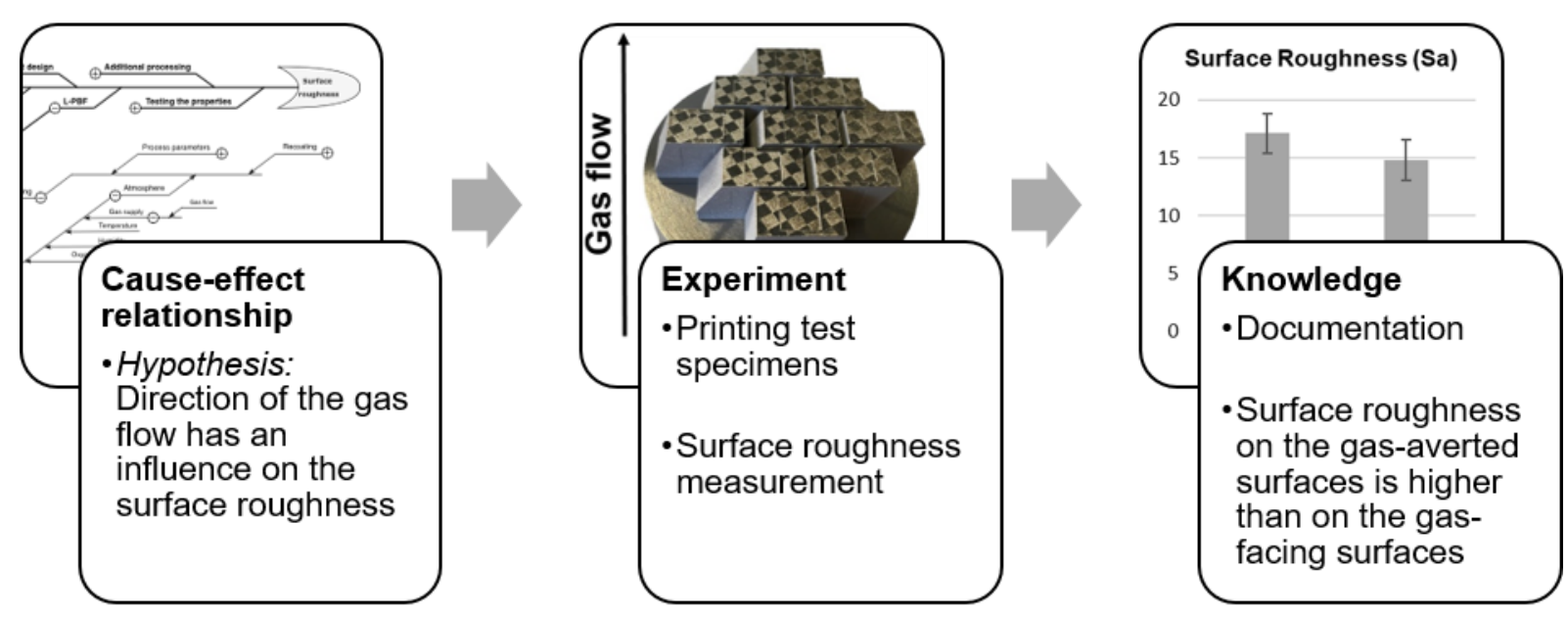

Figure 6. Analysis of sub-causes

\subsection{Determination of solutions and implementation}

With the knowledge gained from the previous steps different solutions were generated. The determined solutions can relate to both the part design and the manufacturing process. In the following, one solution is explained in more detail:

Insert material allowance for function-relevant surfaces in the part design, which consider the gas flow direction.

For this guideline, it is necessary to have an overview of the different process steps. It is important to know the position on the build plate as well as the surface finishing technology. Some technologies, like electro polishing, depend on the surface roughness. In this case, the knowledge about the higher surface roughness on gas-averted surfaces can be taken into account when defining the amount of material allowance. Finally, this design guideline can be considered for future designs and the associated defect can be avoided. As a result, iteration steps due to insufficient surface quality can be reduced.

\section{DISCUSSION}

One challenge of the presented approach is the complexity and the many correlations. It is important to save the results of the individual approach steps in a structured and clear manner. Only in this way, it is possible to find specific solutions to reduce iterations in the production of spare parts.

\section{Identification of defects and correlations between defects and properties}

In already existing defect catalogues, only the most frequently occurring defect types and their effects on a component are considered (Schlauf et al., 2019). However, since spare parts are individual parts, a variety of defects can occur due to different geometries. For this reason, all possible defects should be taken into account during the identification and graded according to the probability of occurrence. The gradation serves to prioritise the defects in further steps of the approach.

The study shows that an L-shaped matrix diagram is a suitable method to safe the results of the two steps. Although this diagram made it possible to illustrate the relationships between the defects and properties clearly, it did not record the relationships between the defects and the properties among themselves. By extending this matrix to a Multiple-Domain Matrix (MDM) (Lindemann et al., 2009), 
all partial results of the first two steps, including the correlations, can be clearly visualised. In addition, the crosses in the diagram can be replaced by circles. Depending on whether the circle is not filled, half-filled or filled, the grade of influence can be also visualised. Alternatively, upwards or downwards facing arrows can be used to show a positive or negative influence with the inclination angle as a measure for the relation strength (Weber et al., 2020a).

\section{Cause-effect relationships}

The Ishikawa-Diagram was found to give a good overview of cause-effect relationships. To tackle all previously identified defects, a separate diagram has to be created for each defect. The gradation of the defects according to the probability of occurrence determines the order, in which the defects should be processed. Due to the organised structure, in which the process steps are the main causes, a quick adaption for other defects is possible. Furthermore, the causes of defects can be compared quickly. Nevertheless, it is not possible to show the correlations between the causes in this diagram. A network model can give an alternative presentation of such correlations.

\section{Analysis of sub-causes}

Not every cause can be controlled by the part design with the aim of eliminating the effect. For the laser power as a sub-cause for example, the effect cannot be influenced by following design guidelines. Therefore, it is mandatory to determine the sub-causes that can be addressed by part design, e.g. gas flow, first and to analyse the exact cause-effect relationship in a subsequent step.

\section{Determination of solutions and implementation}

Apart from design guidelines, there are more solutions for iteration reduction, which are based on cause-effect relationships. Three concepts for iteration reduction in spare part production are defined in the following:

\section{Avoiding defects}

This concept is considered as the preferred option. It involves finding solutions that eliminate the cause of the defects. One possibility is to examine the process step, in which the error occurs and to integrate specific solutions into this process step. The other possibility is to determine the preceding process steps and to find solutions, which are integrated into a preceding process step. In this way, the defect can be eliminated or handled in an early stage. For the latter possibility, the superordinate relationships have to be considered.

\section{Detection of defects}

This concept can be used, if the first concept is not applicable. In this case, detection methods are determined, which in the best case recognise the defect as soon as it occurs.

On the one hand, early detection gives the possibility to terminate the process immediately. So the subsequent process steps are no longer completed and time can be saved. On the other hand additional work steps can be integrated in the following process steps to compensate the defect. Examples of such detection methods in the L-PBF process are in-situ monitoring systems.

\section{Correction of defects}

If the causes of the defects are unavoidable at an early stage, there is still the option of compensating these defects in a later process step. In combination with the second concept - the early detection of defects - additional work steps can be integrated in a later process step. In this way, defects can be compensated so that the required properties are still achieved. For example, if it is known, that large pores occur, hot isostatic pressing (HIP) can be applied for closing them (Du Plessis and Macdonald, 2020).

The first and third concept can be implemented through appropriate design. For all remaining causes, for which no design solutions are possible, other methods have to be applied.

\section{CONCLUSION AND OUTLOOK}

For small batch sizes as they are used for spare parts, producing the part at the first attempt is very important. In this context, additive manufacturing technologies, such as L-PBF, are mentioned, 
because they are often associated with unlimited design freedom. If it is possible to improve the spare part by the use of AM, the potential of the manufacturing process can be exploited. However, as spare parts have to fulfil a functionality, which is defined by geometrical dimensions, fully exploiting the potential of AM is not always possible. Especially for these cases, it is necessary to comprehend the manufacturing process and to identify the different correlations within the entire process. With this preliminary work, limitations of the process can be revealed and considered in early process steps. The complexity of the entire process is a major challenge in identifying such limitations by means of cause-effect relationships.

In this study, a structured approach was presented, to reduce iteration steps systematically without the trial-and-error approach. An example was shown, where on the one hand, partial aspects of causeeffect relationships were examined in detail and on the other hand, the interrelationships in the entire process were considered to generate solutions, which can be implemented in the part design. In order to reduce the number of iterations in the production of spare parts further, other solutions that cannot be implemented by the design will be considered in the future.

This approach is also important for the production of individualised implants. The geometrical dimensions have to be adapted to the body. Thus, the geometry not always can be optimised for AM and the limitations of the process must be known and considered to reduce iteration steps in the manufacturing process.

Further, the properties of an AM part can vary from machine to machine. Thus, it is important to know the limitations of an individual AM system. This approach helps to identify the relations within the AM system. In this way, repeating tests can be avoided and time can be saved.

Nevertheless, there is still a demand for further research on suitable methods, which can be implemented in the individual approach steps and present the relationships clearly.

\section{REFERENCES}

Beiderbeck, D., Krüger, H. and Minshall, T. (2020), "The Future of Additive Manufacturing in Sports”, in Schmidt, S.L. (Ed.), 21st Century Sports, Future of Business and Finance, Springer International Publishing, Cham, pp. 111-132.

Di Wang, Yang, Y., Yi, Z. and Su, X. (2013), "Research on the fabricating quality optimization of the overhanging surface in SLM process", The International Journal of Advanced Manufacturing Technology, Vol. 65 No. 9-12, pp. 1471-1484. 10.1007/s00170-012-4271-4.

Diegel, O., Nordin, A. and Motte, D. (2019), A Practical Guide to Design for Additive Manufacturing, Springer Singapore, Singapore. 10.1007/978-981-13-8281-9.

Du Plessis, A. and Macdonald, E. (2020), "Hot isostatic pressing in metal additive manufacturing: X-ray tomography reveals details of pore closure", Additive Manufacturing, Vol. 34, p. 101191. 10.1016/j.addma.2020.101191.

Frandsen, C.S., Nielsen, M.M., Chaudhuri, A., Jayaram, J. and Govindan, K. (2020), "In search for classification and selection of spare parts suitable for additive manufacturing: a literature review", International Journal of Production Research, Vol. 58 No. 4, pp. 970-996. 10.1080/00207543.2019.1605226.

Fraunhofer IAPT (2020), And suddenly the printed wheel carrier brakes..., available at: https://www.iapt.fraunhofer.de/en/press-media/Press_releases/Press_Release_Fiat_Chrysler.html.

Jakoby, W. (2019), Qualitätsmanagement für Ingenieure, Springer Fachmedien Wiesbaden, Wiesbaden. 10.1007/978-3-658-26596-0.

Kasperovich, G. and Hausmann, J. (2015), "Improvement of fatigue resistance and ductility of TiAl6V4 processed by selective laser melting", Journal of Materials Processing Technology, Vol. 220, pp. 202-214. 10.1016/j.jmatprotec.2015.01.025.

Kranz, J., Herzog, D. and Emmelmann, C. (2015), "Design guidelines for laser additive manufacturing of lightweight structures in TiAl6V4", Journal of Laser Applications, Vol. 27 No. S1, S14001. 10.2351/1.4885235

Li, B.-Q., Li, Z., Bai, P., Liu, B. and Kuai, Z. (2018), "Research on Surface Roughness of AlSi10Mg Parts Fabricated by Laser Powder Bed Fusion", Metals, Vol. 8 No. 7, p. 524. 10.3390/met8070524.

Lindemann, U., Maurer, M. and Braun, T. (2009), "Modeling the Multiple-Domain Matrix", in Lindemann, U., Maurer, M. and Braun, T. (Eds.), Structural complexity management: An approach for the field of product design, Springer-Verlag Berlin Heidelberg, Berlin, Heidelberg, pp. 67-78.

Maamoun, A.H., Xue, Y.F., Elbestawi, M.A. and Veldhuis, S.C. (2018), "Effect of Selective Laser Melting Process Parameters on the Quality of Al Alloy Parts: Powder Characterization, Density, Surface Roughness, and Dimensional Accuracy", Materials (Basel, Switzerland), Vol. 11 No. 12. 10.3390/ma11122343. 
Mertens, R., Clijsters, S., Kempen, K. and Kruth, J.-P. (2014), "Optimization of Scan Strategies in Selective Laser Melting of Aluminum Parts With Downfacing Areas”, Journal of Manufacturing Science and Engineering, Vol. 136 No. 6. 10.1115/1.4028620.

Montero, J., Weber, S., Bleckmann, M. and Paetzold, K. (2020), “A methodology for the decentralised design and production of additive manufactured spare parts", Production \& Manufacturing Research, Vol. 8 No. 1, pp. 313-334. 10.1080/21693277.2020.1790437.

Schlauf, T., Lutter-Günther, M. and Rosenkranz, C. (2019), Project report-AM 4 Industry - LBM Additive Manufacturing Defect Catalogue.

Shinde, M.S. and Ashtankar, K.M. (2017), “Additive manufacturing-assisted conformal cooling channels in mold manufacturing processes", Advances in Mechanical Engineering, Vol. 9 No. 5, 168781401769976. $10.1177 / 1687814017699764$.

Stef, J., Poulon-Quintin, A., Redjaimia, A., Ghanbaja, J., Ferry, O., Sousa, M. de and Gouné, M. (2018), "Mechanism of porosity formation and influence on mechanical properties in selective laser melting of Ti6Al-4V parts", Materials \& Design, Vol. 156, pp. 480-493. 10.1016/j.matdes.2018.06.049.

Thomas, D.J. (2009), “The Development of Design Rules for Selective Laser Melting”, Thesis, University of Wales Institute, Cardiff, 2009.

Weber, S., Montero, J., Bleckmann, M. and Paetzold, K. (2020a), "Parameters on support structure design for metal additive manufacturing”, Proceedings of the Design Society: DESIGN Conference, Vol. 1, pp. 11451154. 10.1017/dsd.2020.14.

Weber, S., Montero, J., Petroll, C., Schäfer, T., Bleckmann, M. and Paetzold, K. (2020b), “The Fracture Behavior and Mechanical Properties of a Support Structure for Additive Manufacturing of Ti-6Al-4V", Crystals, Vol. 10 No. 5, p. 343. 10.3390/cryst10050343.

Zaeh, M.F. and Branner, G. (2010), "Investigations on residual stresses and deformations in selective laser melting”, Production Engineering, Vol. 4 No. 1, pp. 35-45. 10.1007/s11740-009-0192-y. 